\title{
Theoretical Expectations for Clump Red Giants as Distance Indicators
}

\author{
Léo Girardi \\ Dipartimento di Astronomia, Vicolo dell'Osservatorio 5, I-35122 \\ Padova, Italy
}

\begin{abstract}
Variations of $\sim 0.4$ mag are expected in the $I$-band absolute magnitude of red clump giants, $M_{I}^{\mathrm{RC}}$, as a function of both stellar age and metallicity. This is the case regardless of some potential theoretical uncertainties. The quite large differences in mean ages and metallicities of clump stars among galaxies result in systematic changes (of up to $\sim 0.4 \mathrm{mag}$ ) in their $M_{I}^{\mathrm{RC}}$. These numbers also indicate a distance to the LMC that is not necessarily "short".
\end{abstract}

\section{Introduction}

Any measurement of distance modulus using red clump stars requires the determination of four quantities:

$$
(m-M)_{0}=I^{\mathrm{RC}}(\text { galaxy })-M_{I}^{\mathrm{RC}}(\text { Hipp })-A_{I}+\Delta M_{I}^{\mathrm{RC}} .
$$

The first two - the apparent clump magnitude in an external galaxy, $I^{\mathrm{RC}}$ (galaxy), and its absolute magnitude in the Solar Neighbourhood sampled by Hipparcos, $M_{I}^{\mathrm{RC}}$ (Hipp) - can be accurately measured, with $1 \sigma$ errors typically $<0.03$ mag (e.g. Paczyński \& Stanek 1998). Determinations of the total absorption, $A_{I}$, are often controversial at the level of about $0.2 \mathrm{mag}$, however, as demonstrated by the case of the LMC field population (Udalski et al. 1998; Romaniello et al. 1999; Zaritsky 1999). Even more controversial is the assessment of the intrinsic differences in magnitude between Hipparcos and the external populations of clump stars, $\Delta M_{I}^{\mathrm{RC}}$. In this regard, the dependence of $M_{I}^{\mathrm{RC}}$ on age and metallicity has been claimed to be either (i) small and empirically-calibrated (Udalski 1998a,b, 2000), or (ii) more significant as suggested by stellar models (Cole 1998; Girardi et al. 1998) and observations of open clusters (Twarog, Anthony-Twarog \& Bricker 1999; Sarajedini 1999). Moreover, Girardi \& Salaris (2000) conclude that present empirical calibrations for $\Delta M_{I}^{\mathrm{RC}}$ (Udalski 1998a,b, 2000) do not represent general relations, and hence are not suitable for being used in Eq. (1). The discussion about $A_{I}$ and $\Delta M_{I}^{\mathrm{RC}}$ is especially important in the case of the LMC, because the red clump method has been claimed to provide strong evidence for a "short" distance scale (Udalski 2000, and references therein).

In the limited space of this review, I just recall the main reasons why theoretical models predict non-negligible (up to $0.4 \mathrm{mag}$ ) $\Delta M_{I}^{\mathrm{RC}}$ values. For a detailed discussion - based on the same kind of analysis as here - of the related observational data, I refer to Girardi \& Salaris (2000). 

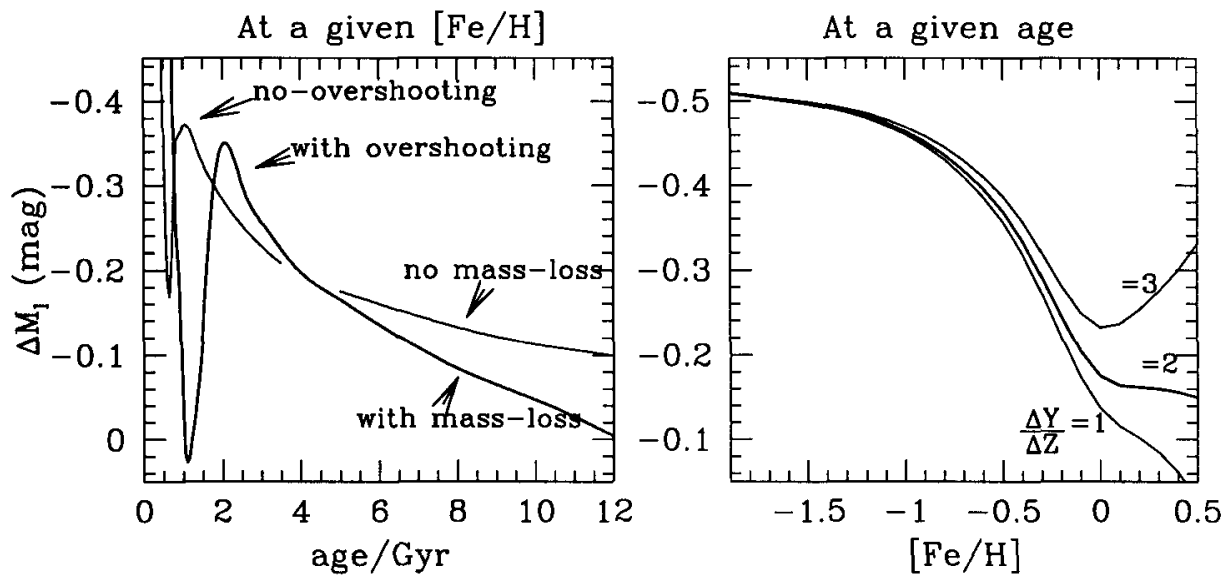

Figure 1. $\quad M_{I}^{\mathrm{RC}}$ as a function of age and $[\mathrm{Fe} / \mathrm{H}]$ (schematic).

\section{Why $M_{I}^{\mathrm{RC}}$ changes with age and metallicity}

Firstly, I recall that in the interval of effective temperatures that characterize red clump stars ( $3.8 \lesssim T_{\text {eff }} \lesssim 3.6$; see Girardi et al. 1998), bolometric corrections in the $I$-band are almost constant (to within $0.1 \mathrm{mag}$ ) and depend very little on metallicity. This means that $M_{I}^{\mathrm{RC}}$ reflects very well the behaviour of $\log L$, a quantity directly predicted by stellar evolution models.

Stellar models have long since predicted that core He-burning stars $(\mathrm{CHeB})$ of low-mass (say $M<2 M_{\odot}$ ), for a given metallicity, should cover a small interval of $\log L$ - as pointed out by e.g. Cannon (1970) and Castellani, Chieffi \& Straniero (1992) - as a consequence of the similar core masses $M_{\text {core }}$ that they have at the He-flash. In fact, similar $M_{\text {core }}$ values imply nearly the same luminosities coming from their He-burning cores, i.e. $L_{\mathrm{He}} \approx$ const. However, it has not been much emphasized that, for the same $M_{\text {core }}$ and metallicity, the luminosity of the H-burning shell increases monotonically with the envelope mass, so that $L_{\mathrm{H}} \propto M$ (roughly). Since $L=L_{\mathrm{H}}+L_{\mathrm{He}}$ with $L_{\mathrm{H}} \approx 2 L_{\mathrm{He}}$ at $M=1 M_{\odot}$, it follows that low-mass clump stars must become some tenths of a magnitude brighter at larger masses/smaller ages. Another trend is that $L$ should decrease with $[\mathrm{Fe} / \mathrm{H}]$, due to the decrease of both $M_{\text {core }}$ and the efficiency of the H-burning shell.

Some $\mathrm{CHeB}$ intermediate-mass stars do also occupy the clump region of the HR diagram (Girardi 1999). Their luminosities change a lot with age, reflecting mainly the proportionality between the core mass at He-ignition and the initial mass, that is followed by stars that do not develop $\mathrm{e}^{-}$-degenerate He cores.

These properties are shared by most (if not all) sets of evolutionary tracks of $\mathrm{CHeB}$ stars present in the literature (e.g. Pols et al. 1998; Charbonnel et al. 1996; Girardi et al. 2000, among others), and are schematically presented in Fig. 1: $M_{I}^{\mathrm{RC}}$ is expected to change by as much as $\sim 0.4 \mathrm{mag}$ with age at a fixed $[\mathrm{Fe} / \mathrm{H}]$, and by $\sim 0.4 \mathrm{mag}$ with $[\mathrm{Fe} / \mathrm{H}]$ at a fixed age. A few theoretical uncertainties (e.g. overshooting, mass-loss and the helium-to-metal enrichment 

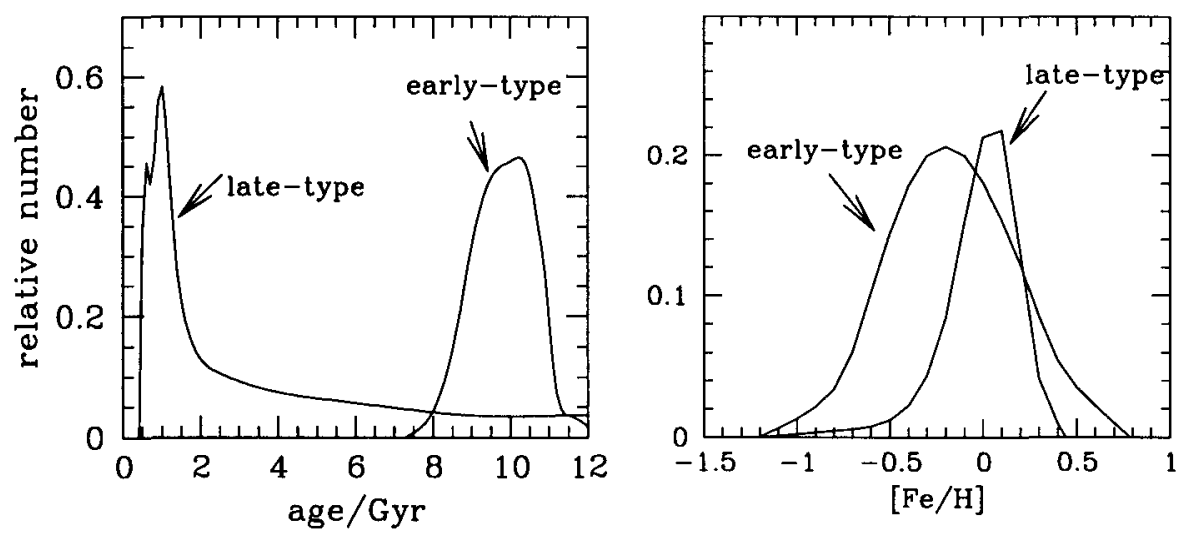

Figure 2. Age and $[\mathrm{Fe} / \mathrm{H}]$ distributions for clump stars in late- and early-type galaxies (schematic).

ratio, as shown in Fig. 1) can change the details of this behaviour by $\sim 0.1 \mathrm{mag}$, but cannot change the general trends.

\section{Why $M_{I}^{\mathrm{RC}}$ varies among galaxies}

Assessing the changes of $M_{I}^{\mathrm{RC}}$ with age and metallicity is only half of the problem in determining the $\Delta M_{I}^{\mathrm{RC}}$ values to be used in Eq. (1). The other half corresponds to answering "What are the age and metallicity distributions of clump stars in galaxies?" This question finds quantitative answers from basic population synthesis theory, once we know the past star formation rate (SFR) and age-metallicity relation (AMR) of the galaxies considered (see Girardi \& Salaris 2000). Fig. 2 shows two illustrative cases:

(i) In a late-type galaxy that has formed stars at a nearly constant rate since its formation (e.g. the disks of spirals, or irregulars), the age distribution of clump stars peaks at relatively young ages $(1-3 \mathrm{Gyr})$. This occurs because: (i) the rate at which stars leave the main sequence decreases with age; and (ii) the He-burning lifetime has a maximum for stars of $\sim 1$ Gyr. Notice that a predominantly young clump implies a rather narrow $[\mathrm{Fe} / \mathrm{H}]$ distribution, since, in general, galaxies have experienced little chemical evolution in the last few gigayears.

(ii) In an early-type galaxy where star formation ceased after just a few Gyr (e.g. ellipticals and the bulges of spirals), clump stars younger than $\sim 8$ Gyr simply do not exist and the clump age distribution is narrow. These old populations, in general, present a broader distribution of $[\mathrm{Fe} / \mathrm{H}]$ values.

Of course, any intermediate (and even more extreme) behaviour can occur. The general situation is that quite different distributions of ages/metallicities of clump stars are expected among different galaxies. The local sample of stars that define the Hipparcos clump, is also expected to be quite different from those found in other Local Group systems. 


\section{Conclusions about $\Delta M_{I}^{\mathrm{RC}}$}

If we consider Figs. 1 and 2 together, the conclusions are almost immediate: non-negligible values of $\Delta M_{I}^{\mathrm{RC}}$ are expected among different galaxies. Girardi et al. (1998) and Girardi \& Salaris (2000) find $\Delta M_{I}^{\mathrm{RC}}$ values as large as $0.2-$ 0.3 mag for the Magellanic Clouds. When one uses these theoretical population corrections, and the Romaniello et al. (1999) or Zaritsky (2000) determinations for $A_{I}$, it turns out that the red clump method is still compatible with a "long" distance scale, with $(m-M)_{0}^{\mathrm{LMC}}=18.55 \pm 0.05 \mathrm{mag}$ for the LMC.

These conclusions seem to be in contradiction with Udalski (1998a,b, 2000), who, on observational grounds, finds a small dependence of $M_{I}^{\mathrm{kC}}$ on both age and metallicity. However, Girardi \& Salaris (2000) clarify that (i) larger dependences, compatible with theoretical models, are found by Twarog et al. (1998) and Sarajedini (1999); (ii) Udalski's empirical relations do not have general validity, since they reflect the particular distributions of ages and metallicities of clump stars included in the observational samples, and express the clump behaviour by means of an over-simplified relation between $M_{I}^{\mathrm{RC}}$ and $[\mathrm{Fe} / \mathrm{H}]$ (which, cf. Figs. 1 and 2, is not expected to be satisfactory, for both star clusters and galaxies). According to Girardi \& Salaris, theoretical models still provide the most reliable $\Delta M_{I}^{\mathrm{RC}}$ values, provided that the SFR and AMR of the galaxies under scrutiny are sufficiently well known.

In summary, theoretical predictions seem to survive most tests provided by observational data (cf. Girardi \& Salaris 2000), and yet lead to an interpretation of the data that is substantially different - and much more complete! - than the one sketched in present empirical $M_{I}^{\mathrm{RC}}$ versus $[\mathrm{Fe} / \mathrm{H}]$ calibrations. Can we really do without theoretical models?

Acknowledgements I am greatly indebted to Maurizio Salaris, Martin Groenewegen and Achim Weiss, my collaborators in the clump work.

\section{References}

Cannon, R.D. 1970, MNRAS, 150, 111

Castellani, V., Chieffi, A., \& Straniero, O. 1992, ApJS, 78, 517

Charbonnel, C., Meynet, G., Maeder, A., \& Schaerer, D. 1996, A\&AS, 115, 339

Cole, A.A. 1998, ApJ, 500, L137

Girardi, L. 1999, MNRAS, 308, 818

Girardi, L., Groenewegen, M.A.T., Weiss, A., \& Salaris, M. 1998, MNRAS, 301, 149

Girardi, L., \& Salaris, M. 2000, MNRAS, in press.

Girardi, L., Bressan, A., Bertelli, G., \& Chiosi, C. 2000, A\&AS, 141, 371

Paczyński, B., \& Stanek, K.Z. 1998, ApJ, 494, L219

Pols, O.R., et al. 1998, MNRAS, 298, 525

Romaniello, M., Salaris, M., Cassisi, S., \& Panagia, N. 2000, ApJ, 530, 738

Sarajedini, A. 1999, AJ, 118, 2321 
Twarog, B.A., Anthony-Twarog, B.J., \& Bricker A.R. 1999, AJ, 117, 1816

Udalski, A. 1998a, Acta Astr., 48, 113

Udalski, A. 1998b, Acta Astr., 48, 383

Udalski, A. 2000, ApJ, 531, L25

Udalski, A., et al. 1998, Acta Astr., 48,1

Zaritsky, D. 1999, AJ, 118, 2824 